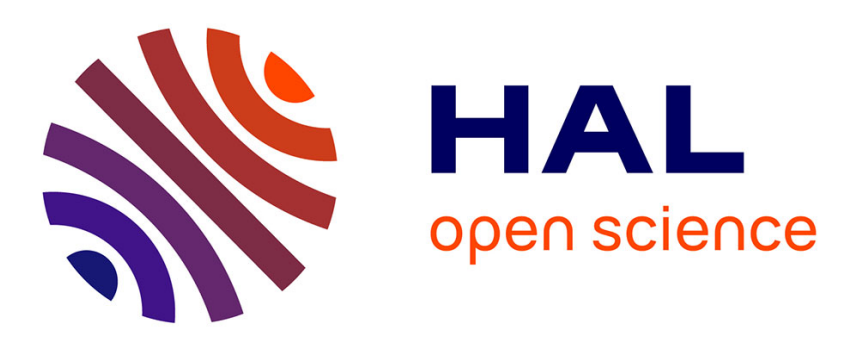

\title{
Empirical Mode Decomposition (EMD) for multi-gate, multi-transducer Ultrasound Doppler fetal heart monitoring
}

Denis Rouvre, Denis Kouamé, François Tranquart, Léandre Pourcelot

\section{To cite this version:}

Denis Rouvre, Denis Kouamé, François Tranquart, Léandre Pourcelot. Empirical Mode Decomposition (EMD) for multi-gate, multi-transducer Ultrasound Doppler fetal heart monitoring. 5th IEEE International Symposium on Signal Processing and Information Technology (ISSPIT 2005), Dec 2005, Athène, Greece. pp.208-212, 10.1109/ISSPIT.2005.1577097 · hal-03149789

\author{
HAL Id: hal-03149789 \\ https://hal.science/hal-03149789
}

Submitted on 24 Feb 2021

HAL is a multi-disciplinary open access archive for the deposit and dissemination of scientific research documents, whether they are published or not. The documents may come from teaching and research institutions in France or abroad, or from public or private research centers.
L'archive ouverte pluridisciplinaire HAL, est destinée au dépôt et à la diffusion de documents scientifiques de niveau recherche, publiés ou non, émanant des établissements d'enseignement et de recherche français ou étrangers, des laboratoires publics ou privés. 
2005 IEEE International

Symposium on Signal Processing

and Information Technology

\title{
Empirical Mode Decomposition (EMD) for multi-gate, multi-transducer Ultrasound Doppler fetal heart monitoring
}

\author{
Denis Rouvre ${ }^{1}$, Denis Kouamé ${ }^{1}$, François Tranquart ${ }^{2}$, and Léandre Pourcelot ${ }^{1}$ \\ ${ }^{1}$ LUSSI CNRS FRE 2448, Université de Tours, Faculté de Médecine, 10 boulevard Tonnelé, 37032 Tours Cedex 1, France. \\ E-mail: kouame@univ-tours.fr \\ ${ }^{2}$ Inserm U619, Hopital Bretonneau-CHRU Tours, 37044 Tours cedex 9, France. E-mail: tranquart@univ-tours.fr
}

\begin{abstract}
This paper presents a new technique called Empirical Mode Decomposition (EMD) applied to a multi-gate, multitransducer Ultrasound Doppler system used for fetal heart monitoring. We propose this system as an alternative to the existing fetal monitoring techniques.

Classical autocorrelation-based Fetal Heart Rate (FHR) detection has been shown to be a good method to detect the FHR in normal situations. However, as this method is applied to magnitudes of the Doppler signal fails to estimate the Fetal Heart Rate when the fetus moves. In view of the extent of FHR variability, a monitoring system should be able to estimate this parameter each time. We therefore propose empirical mode decomposition as an interesting alternative for long-term monitoring.

The principle of this method consists of iterative decompositions of a signal into a sum of functions that have the same number of extrema, the same number of zero crossings, and are symmetric with respect to the local mean. When investigation of FHR using autocorrelation on the original Doppler signal fails due to fetal movement or low Signal-to-Noise Ratio (SNR), it is frequently successful using the intrinsic mode functions $(i m f s)$.

We compared the results of multi-transducer FHR detection with and without EMD decomposition using in-vivo Doppler signals from a set of 40 women between 32 and 38 weeks of pregnancy. This method greatly improved the quality of FHR detection.
\end{abstract}

Index Terms-Empirical Mode Decomposition, Electronic fetal monitoring, Actifoetus

\section{INTRODUCTION AND REQUIREMENTS FOR FETAL MONITORING}

There are several different types of fetal monitoring systems. Ultrasound B-mode scanners are used to obtain fetal measurements in order to detect abnormal growth, and Doppler measurements are used to study blood circulation between the mother and fetus and also in the umbilical cord. All these measurements are performed at the beginning of the pregnancy and periodically during the pregnancy. Another type is a continuous or discontinuous electronic fetal heart rate monitoring device, the cardiotocogram, which is the reference in the field of Electronic Fetal Monitoring (EFM) [1] but this only measures the FHR and not fetal movements. Ultrasound (US) Doppler provides both detection of the FHR and fetal movements of the fetus, thus providing more information on fetal well-being, and is widely used for FHR monitoring. Whether mono or bi-transducer, these systems using continuous or pulsed ultrasound waves provide partial automated detection of movements [2] and fetal breathing [3] [4].

The aim of this study was to develop a more complete system consisting of a multi-transducer multi-gate prototype device with specific signal processing techniques for fetal mon- itoring to form a fetal biophysical profile providing more information on fetal behavior than FHR alone [5].

Moreover, with data from 40 women between 32 and 38 weeks of pregnancy, each carried out with the Actifoetus system, we showed that classical autocorrelation based Fetal Heart Rate (FHR) detection is not always satisfactory, and we have developed a new signal processing technique based on Empirical Mode Decomposition (EMD) to improve such detection. We focus here on FHR detection.

In a first part of this article, we present our acquisition device, referred to as Actifoetus. In the second part we introduce the new signal processing method called Empirical Mode Decomposition, and finally we show in-vivo results to illustrate the value of the system.

\section{MATERIALS}

\section{A. The system}

A new Doppler system, referred to as Actifœtus, has been developed for fetal activity monitoring (Actifœtus system built by Ultrasons Technologies ${ }^{\mathrm{TM}}$ ). It consists of twelve $2 \mathrm{MHz}$ transducers (3 groups of 4 transducers) with five gates. The Actifoetus system comprises a Pentium 42 Ghz based Personal Computer, dedicated management software, an electrical security transformer in order to comply with safety requirements in the hospital, and the prototype electronic device. This device includes three electronic pulsed Doppler electronic Boards, a data acquisition board, and three groups of transducers. The emission signal is sinusoid, with frequency $f_{0}=2 M h z$. The received signal is first amplified by a lownoise amplifier, then by a deep compensated amplifier in order to compensate for the strong attenuation of the deepest gates. Finally the signal is band pass filtered (center frequency $f_{0}$ ). After complex in-phase demodulation, the components are sampled sequentially and converted. Pulse Repetition Frequency (PRF) is $1 k H z$. Data are recorded directly on the computer hard drive.

Body exploration is performed with all twelve transducers. Adjustable gates ranging from 2 to 14 centimeters are explored and these 5 gates are independently adjustable in depth using software.

\section{B. Transducer arrangement}

In order to obtain a better interpretation of the signals measured, transducers are divided into three groups of four trans208 
support. A transducer group is composed of 4 transducers : a central transducer and 3 transducers placed around the central transducer. Fig. 2 shows a transducer group arrange-

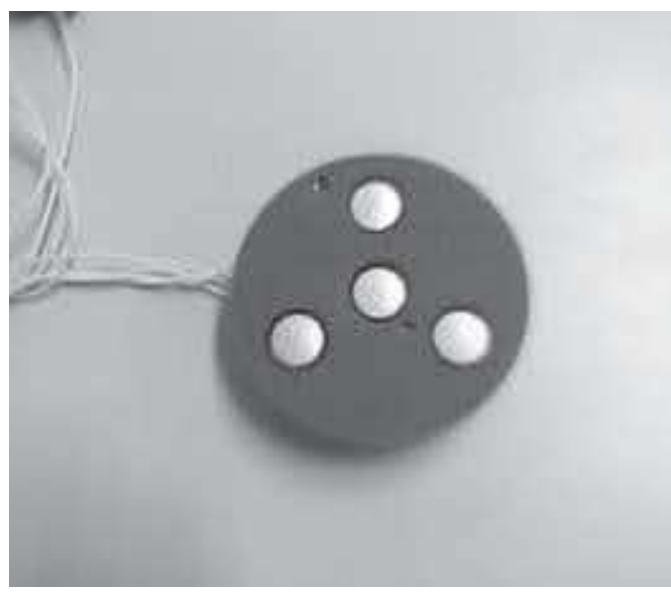

Fig. 1. A transducer group is composed of 4 transducers : a central transducer and 3 transducers placed around the central transducer.

ment during monitoring. Group A (transducers \#1-\#4) explores the fetal thorax, investigating fetal heart rate and breathing movements, Group B (transducers \#5-\#8) the upper limbs, and Group C (transducers \#9-\#12) the lower limbs. The twelve transducers monitor the activity of all the regions of interest in the fetus. The ultrasound beam of the transducers is as wide as possible to maximize the region of exploration. A belt is used throughout the recording in order to prevent transducer movement.

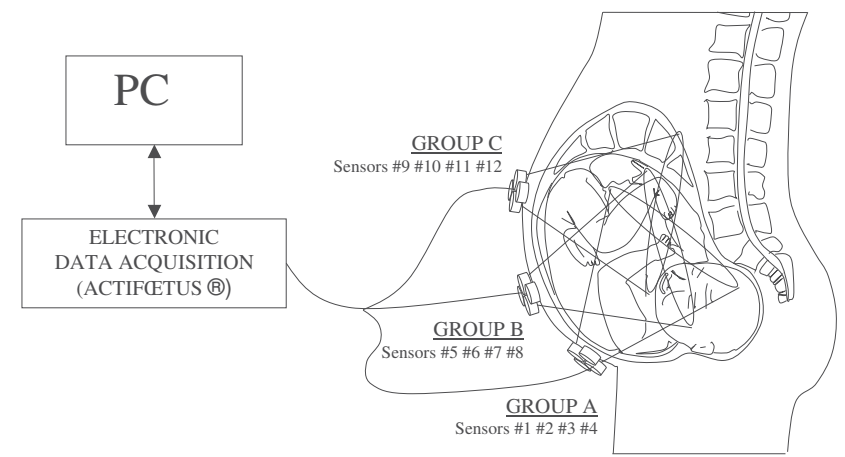

Fig. 2. Transducer group arrangement. Group A (transducers \#1-\#4) explore the fetal thorax, investigating fetal heart rate and breathing movements, Group $B$ (transducers \#5-\#8) the upper limbs and Group C (transducers \#9-\#12) the lower limbs.

\section{SIGNAL PROCESSING BACKGROUND}

Before introducing the empirical mode decomposition processing technique, we briefly summarize the Ultrasound Doppler signal parameters and signal processing techniques used to extract fetal heart rate information.

\section{A. Ultrasound Doppler signal parameters}

Ultrasound (US) Doppler is a widely used method to evaluate the behavior of moving targets in terms of velocity and related information. When an ultrasound wave with frequency $f_{0}$ is emitted from a transducer towards a moving target, the frequency of the backscattered wave received is $f_{0}+f_{d}$. After complex in-phase and in-quadrature demodulation, the analytical Doppler signal can be written as :

$$
y(t)=K(t) \exp j\left[2 \pi f_{d}(t)+\varphi(t)\right]=I(t)+j Q(t)
$$

where $K(t)$ is the random magnitude of the Doppler signal, depending on the characteristics of the transducers and the measurement area, and $\varphi(t)$ is the random phase, depending on the positions of the scatterers in the flowing fluid. The frequency shift $f_{d}$ (called Doppler frequency) may be written as :

$$
f_{d}(t)=\frac{2 f_{0}}{c} \cos \theta v(t)
$$

where $v=|\vec{v}|$ and $\vec{v}$ is target velocity, $\theta$ is the angle between the ultrasound beam and vector $\vec{v}$, and $c$ is the ultrasound wave velocity in the medium. Angle $\theta$ does not have to be evaluated precisely here, since the purpose is to extract variations in fetal heart rate.

This Doppler signal contains a large amount of information concerning target movement (velocity, position) via the Doppler frequency (2) and the overall phase, which can be obtained by (3), expresses the distance of the transducer from the scatterers in millimeters, with a factor $\frac{4 \pi}{\lambda}$.

$$
\phi=\arctan \left(\frac{Q(t)}{I(t)}+n \pi\right) .
$$

The velocity of the target, which is proportional to Doppler frequency, can be accessed by the time frequency distribution [6],[7].

Finally, the magnitude of the backscattered signal depends on the characteristics of the transducers and the nature of the scatterers. A direction separation filter is necessary to follow the magnitude of movements towards and away from the transducer separately. This eliminates both positive frequency components and negative frequency components. This separation filter thus gives two signals (forwards and backwards). As mentioned above, direction information can also be accessed through the overall phase.

\section{B. Detecting fetal heart rate using Doppler signal processing}

Initially a convolution algorithm [8], based on three-stage computation using the directional magnitude of the Doppler signals $x=[x(1), \ldots, x(N)]$, was used in order to estimate the FHR. During the first stage, the signal was smoothed. The autoconvolution

$$
R_{x}(p)=\sum_{p=0}^{P-1} x(n-p) x(n-N+p)
$$

was evaluated and then low-pass filtered (cut-off frequency $20 \mathrm{~Hz}$ ). In the second stage, the intervals between two suc$209^{\text {cessive peaks were evaluated (representing intervals between }}$ 
two successive heartbeats), and in the last stage the results obtained for the two signal magnitudes were compared. When they agreed, these values were validated.

The performance of this algorithm was very poor when the SNR was low, and we therefore investigated a simple alternative to this method, i.e. the autocorrelation method, that estimates the fundamental frequency of a periodic signal, such as a fetal heart Doppler signal. This consists of computing normalized autocorrelation coefficients (5) for $L$ samples of the signal and for lags between $a \times F_{e}$ and $b \times F_{e}$, where $F_{e}$ is the sampling frequency. To allow detection of a rate between 80 to 200 beats per minute (i.e. the typical fetal heart rate range) we set $a=1.33$ beats per second (bps) and $b=3.33 \mathrm{bs}$.

$$
R_{x}(p)=\frac{1}{N} \sum_{n=0}^{N-|p|-1} x(n) x(n+p)
$$

with $p=0, \pm 1, \pm 2, \ldots$

When the maximum autocorrelation coefficient was close to 1 (or greater than an empirical threshold), meaning that the signal was indeed periodic, this coefficient position provided an estimation of the signal period. Note that any time and/or frequency estimator may also be used to estimate the FHR.

Figure 3 show the analysis of a 10 second Doppler signal produced by fetal heart beats for a given transducer at a given gate. In fig.3 (A) we show separate magnitudes (solid and dashed lines), indicating movement towards and away from the transducer. Figure 3, (B) shows the Doppler signal phase, (C) the spectrogram, and (D) the Fetal Heart Rate computed by autoconvolution method and (E) by autocorrelation method. The trend seen in (B) is due to a slight movement of the transducer during the recording.

Although the autocorrelation method outperforms the autoconvolution method, it may also lose the FHR when the signal is noisy. Fig.4 shows an example of a loss of FHR. Fig.4 (A) shows the spectrogram (Time-Frequency diagram) of a transducer signal at a given gate, fig.4 (B) shows the phase of this signal and fig.4 (C) shows the fetal heart rate estimated with the autocorrelation technique. Movement at the $16^{\text {th }}$ second induced a loss of FHR.

\section{Empirical Mode Decomposition}

We have seen that the Autocorrelation FHR detection method is a good method to detect FHR in a normal situation. However, in view of the magnitudes of the signal, this method "looses" the FHR when the fetus is moving (see Fig.4). In view of recognized extent of FHR variability, the system must estimate the FHR all the time. We propose empirical mode decomposition as an interesting alternative for this purpose in long-term monitoring.

A new nonlinear technique, referred to as Empirical Mode Decomposition was first introduced by Huang [9] to represent nonstationary signals as sums of zero-mean Amplitude Modulation (AM) and Frequency Modulation (FM) components. In
(A)

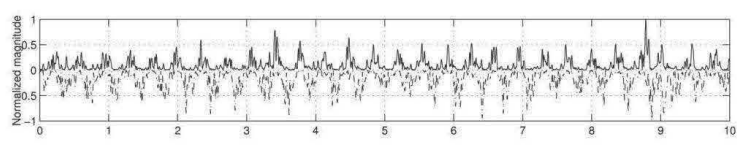

(B)

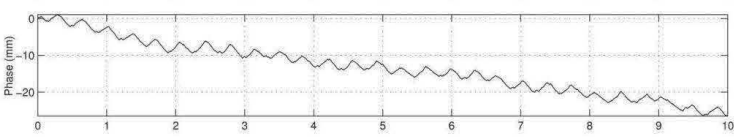

(C)

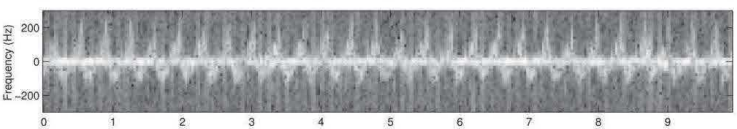

(D)

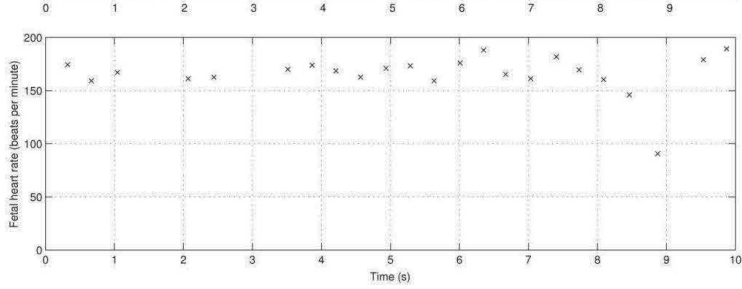

(E)

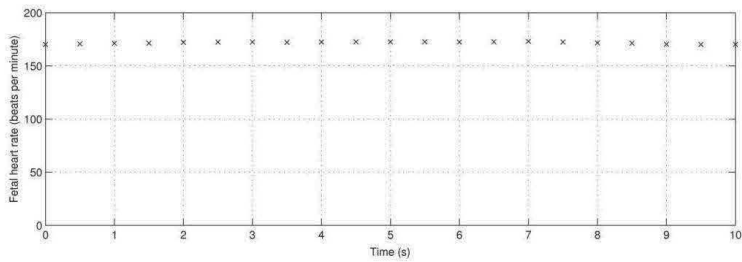

Fig. 3. Analysis of 10 second Doppler signal produced by fetal heartbeats for a given transducer at a given gate. Separated magnitudes (solid and dashed lines) are movements towards and away from the transducer, respectively (A), Doppler signal phase (B), spectrogram (C), Fetal Heart Rate computed by autoconvolution method (D) and by autocorrelation method (E). The trend seen in (B) is due to a slight movement of the transducer during the recording.

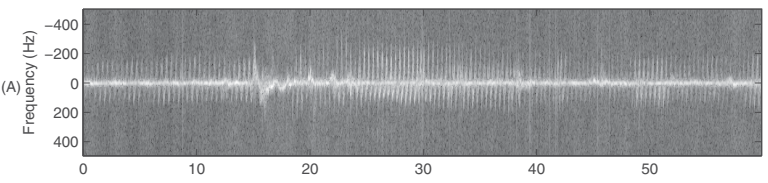

(B)
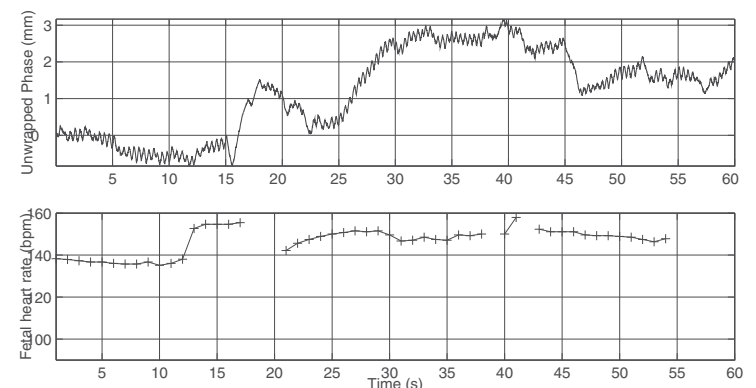

Fig. 4. Analysis of 60 second Doppler signal produced by fetal heartbeats for a given transducer at a given gate. (A) shows the spectrogram of the transducer signal. (B) shows the phase. (C) shows the fetal heart rate estimated with the autocorrelation technique. The movement that can be seen on (A) and (B) at $16^{\text {th }}$ second induced a loss of Fetal Heart Rate. 
spite of frequently interesting results, this technique is defined only by a algorithm, and does not for the moment set out an analytical formulation which would allow for theoretical analysis and performance evaluation. This method has recently been further explored by Flandrin and Rilling [10], [11], [12], in particular to study its denoising properties.

The principle consists of decomposing a signal $\mathrm{x}(\mathrm{t})$ into a sum of functions that have the same numbers of zero crossings and extrema and are symmetric with respect to the local mean. This method therefore seems to be adaptable to the Doppler signal.

The first situation is similar to the narrow band requirement for a stationary Gaussian process. The second modifies an overall requirement to a local requirement, and is necessary to ensure that the instantaneous frequency will not have unwanted fluctuations as induced by asymmetric waveforms. These functions are called intrinsic mode functions ( $\mathrm{im} \mathrm{fs}$ ). The decomposition is based on the direct extraction of the energy associated with various intrinsic time scales, the most important parameters of the system.

Given a signal $\mathrm{x}(\mathrm{t})$, the algorithm of EMD (called sifting process [9] [10]), can be summarized as follows :

1) identify all extrema of $x(t)$

2) interpolate between minima (resp. maxima), ending up with some envelopes $e_{\min }(t)$, resp. $e_{\max }(t)$

3) compute the mean $m(t)=\left(e_{\min }(t)+e_{\max }(t)\right) / 2$

4) extract the detail $d(t)=x(t)-m(t)$

5) iterate on the residual $m(t)$

In practice, the above procedure must be redefined by a sifting process [9], a method which amounts to first iterating steps 1 to 4 upon the signal detail $\mathrm{d}(\mathrm{t})$, until the latter can be considered as a zero-mean signal according to a stopping criterion. Once this has been achieved, the detail is considered to be the effective $i m f$, the corresponding residual is computed and step 5 applies.

This construction means that the number of extrema decreases from one residual to another. There is thus a finished number of $i m f s$ in the signal. In our case, the application of the EMD amounts to signal filtering with nondefinite frequency bands which depend on the signal characteristics.

\section{IN-VIVO RESULTS}

Clinical validation of the Actifoetus system is in hand at a University Hospital and is being subjected to a clinical protocol. We have meanwhile about 40 measurements of 30 minutes, each carried out on pregnant women with the Actifoetus system after the third trimester echography. The position of the fetus was thus known before recording.

\section{A. Short term measurement}

In fig. $5\left(a_{1}\right)$ a Doppler signal from a pregnant woman can be seen coming from transducer 1 (third depth, between 7 $\mathrm{cm}$ and $9.5 \mathrm{~cm}$ ) of the Actifoetus. This signal consisted of movement and FHR. On this recording, a small movement was noted between the third and the fourth seconds. This movement can be seen on the third imf (imf\#3). Classical autocorrelation-based FHR detection failed on the original signal, because of this movement and of poor SNR. Thus, EMD enabled us to separate the movement from the rhythm in this case, and to detect FHR on the first imf. Fetal heart beat was found on the first $i m f(i m f \# 1$ ), and was $137.6 \mathrm{bpm}$. This corresponded to the $138 \mathrm{bpm}$ measured during recording.

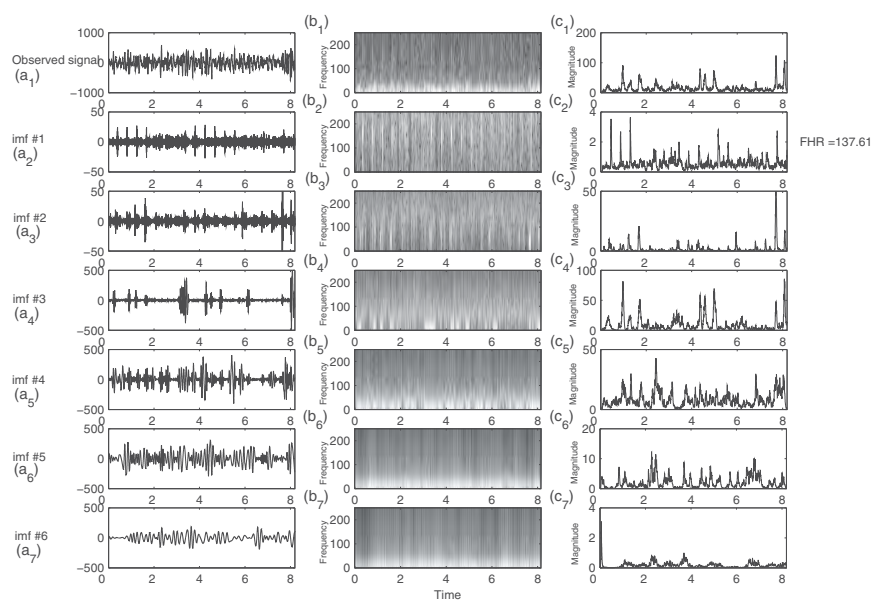

Fig. 5. Analysis of 8 second Doppler signal produced by fetal heartbeats for transducer 1 at the third depth. Empirical Mode Decomposition of an Actifoetus observation signal $\left(a_{1}\right)$. $\left(b_{1}\right)$ represents Time frequency diagram of $\left(a_{1}\right),\left(c_{1}\right)$ represents magnitude of $\left(a_{1}\right) .\left(a_{2}\right)$ represents the first imf of $\left(a_{1}\right),\left(b_{2}\right)$ represents Time frequency diagram of $\left(a_{2}\right),\left(c_{2}\right)$ represents magnitude of $\left(a_{2}\right)$. ( $\left.a_{3}\right)$ represents the second imf of $\left(a_{1}\right)$, etc... Autocorrelation detection is computed on magnitudes, column $(c)$. FHR detection failed on the original signal but succeeded on the first imf, and was $137.6 \mathrm{bpm}$.

It can be seen that that the FHR was detected on one of the first three imfs whereas the movements present in the original signal are visible on imfs higher than three.

\section{B. Long term measurement}

The value of this system was evident with long-term monitoring. As shown on fig. 6, we compared the autocorrelation method applied directly to the signal coming from transducer 1 (third depth, between $7 \mathrm{~cm}$ and $9.5 \mathrm{~cm}$ ) with the autocorrelation method applied to both the imfs and the original signal. In this in-vivo situation, the FHR was mainly detected on $i m f \# 2$ and $i m f \# 3$, whereas detection often failed when not using EMD, mainly due to the presence of noise.

Fig. 6 (c) shows that the FHR detection using Autocorrelation on the imfs greatly improved the quality of FHR detection, compared to the autocorrelation method applied directly to the signal (fig. 6 (b)). In view of the extent of FHR variability, it is clear that the multi-transducer Autocorrelation method can fail to detect the fetal heart rate in the presence of fetal movement or low SNR. EMD can help to separate movement (or MHR) from the FHR signal, thus improving detection. 

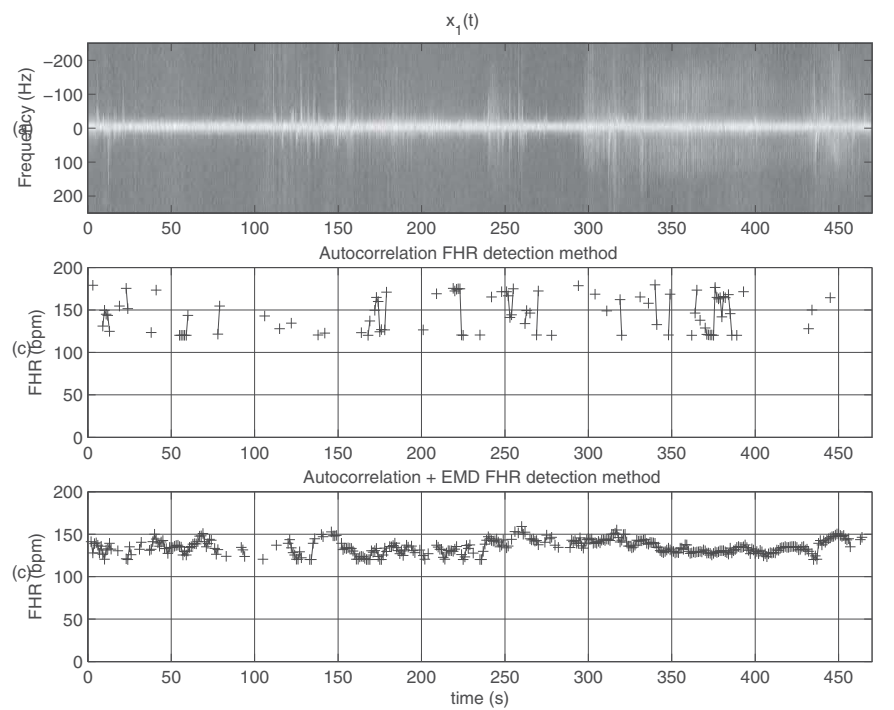

Fig. 6. Analysis of 8 minutes' Doppler signal produced by fetal heartbeats for transducer 1 at the third depth. Spectrograms of Actifoetus transducer signal (a), FHR detection using Autocorrelation method applied directly to the signal (b), FHR detection using Autocorrelation on the imfs (c). Recording duration : $8 \mathrm{~min}$.

\section{DISCUSSION}

We have presented here a new signal processing method called Empirical Mode Decomposition applied to a multi transducer, multi-gate Doppler device for Electronic Fetal Monitoring.

Using Empirical Mode Decomposition, we showed that this method helps to extract the fetal heart signal from one of the intrinsic mode functions ( $\mathrm{imfs}$ ) resulting from Empirical Mode decomposition when the fetal heart signal is present but not extractable from the original source signal. However, this is a new technique which has not yet been completely formalized from a theoretical point of view, and computation time may vary according to intrinsic signal characteristics.

\section{CONCLUSION}

Initial investigations for evaluating fetal heart rate using a multi-transducer, multi-gate system have shown that Empirical Mode Decomposition is a promising method to improve detection of the Fetal Heart Rate. In particular, we have shown that fetal heart rate detection can be improved by applying autocorrelation to the $i m f s$ after Empirical Mode Decomposition. EMD may also help to separate the different sources of the activity such as movements and FHR.

This method will now be investigated to improve detection of pseudo-respiration movements in the fetus, which are still difficult to extract.

\section{REFERENCES}

[1] Royal College of Obstetricians and Gynaecologists, The Use of Electronic Fetal Monitoring, The use and interpretation of Cardiotocography in intrapartum fetal surveillance, RCOG Press, 2001.

[2] B. Karlsonn, M. Berson, T.Helgason, R.T. Geirsson, and L. Pourcelot, "Effect of fetal and maternal breathing on the ultrasonic Doppler signal due to fetal heart movement," European journal of ultrasound, vol. 11, pp. 47-52, 2000.
[3] B. Karlsonn, K. Foulquière, K. Kaluzynski, F. Tranquart, A. Fignon, D. Pourcelot, L. Pourcelot, and M. Berson, "The dopfet system : a new ultrasonic Doppler system for monitoring and characterisation of fetal movement," Ultrasound in Med \& Biol., vol. 26, no. 7, pp. 1117-1124, 2000.

[4] Y. Yamakoshi, T. Shimizu, N. Shinozuka, and H. Masuda, "Automated fetal breathing movement detection from internal small displacement measurement," Biomed. Technik, vol. 41, pp. 242-247, 1996.

[5] FA Manning, LD Platt, and Sipos L., "Antepartum fetal evaluation: Development of a fetal biophysical profile," Am J Obstet Genicol, vol. 136, pp. 787-795, 1980.

[6] D. Kouamé, J-M. Girault, and F. Patat, "High resolution processing techniques for ultrasound Doppler velocimetry in presence of colored noise part 1 : Nonstationary methods," IEEE Trans. Ultrason. Ferro. Frequency. Contr., vol. 50, no. 3, pp. 257-266, 2003.

[7] D. Kouamé, J-M. Girault, J-P. Remenieras J-P. Chemla, and M. Lethiecq, "High resolution processing techniques for ultrasound Doppler velocimetry in presence of colored noise part 2: Multiple phase pipe flow velocity measurement," IEEE Trans. Ultrason. Ferro. Frequency. Contr., vol. 50, no. 3, pp. 267-276, 2003.

[8] Karlsson B., Application de l'effet Doppler ultrasonore à l'investigation des mouvements fotaux. Développement et validation d'un appareil bicapteurs et d'un système d'analyse des mesures, $\mathrm{Ph} . \mathrm{D}$. thesis, François Rabelais university of Tours, 1996.

[9] E. Huang and al., "The empirical mode decomposition and the hilbert spectrum for nonlinear and non stationary time series analysis," 1998, vol. 454, pp. 903-995.

[10] P. Goncalves G. Rilling, P. Flandrin, "On empirical mode decomposition and its algorithms," 2003.

[11] G. Rilling P. Flandrin, P. Goncalves, "Detrending and denoising with empirical mode decompositions," 2004.

[12] P. Goncalves G. Rilling, P. Flandrin, "Empirical mode decomposition, fractional gaussian noise, and hurst exponent estimation," 2005. 